\title{
Probiótico na alimentação de tilápias cultivadas em efluentes de esgotos doméstico tratado
}

\author{
Antônio Hosmylton Carvalho Ferreira'*, João Batista Lopes², \\ Maria de Nasaré Bona de Alencar Araripe², Cleto Augusto Baratta Monteiro², \\ Francisco Teixeira Andrade ${ }^{2}$, Janaina Mitsue Kimpara ${ }^{3}$ \\ 'Universidade Estadual do Piauí, Parnaiba, PI, Brasil \\ 2Universidade Federal do Piaú, Teresina, PI, Brasil \\ ${ }^{3}$ Embrapa Meio Norte, Teresina, Pl, Brasil \\ *Autor correspondente, e-mail: hosmylton@hotmail.com
}

\section{Resumo}

A bioimpedância é um método utilizado para estimar de composição corporal em humanos e animais, caracterizado pela determinação do valor do ângulo de fase. Objetivou-se estimar a condição dos peixes por meio da técnica de bioimpedância elétrica e avaliar o desempenho de tilápias-do-nilo cultivadas em água de esgoto doméstico tratado com a adição de probiótico (Bacillus licheniformis, Bacillus cereus e Bacillus subitillis e leveduras -Saccharomyces cerevisiae e Saccharomyces boulardi) em rações. Foram utilizados 360 alevinos, com peso médio inicial de 2,91 $\pm 0,37 \mathrm{~g}$ e comprimento médio inicial $3,30 \pm 0,27 \mathrm{~cm}$, distribuídos em delineamento inteiramente casualizado, com três tratamentos, seis repetições de 20 peixes. Os tratamentos consistiram de: PRO1 - peixes cultivados em água tratada; RO2 - peixes cultivados em água residuária,; PRO3 peixes cultivados em água residuária + probiótico adicionado na ração. Todos alimentados até a aparente saciedade. As tilápias que estavam submetidas ao desafio sanitário (água residuária) apresentaram ângulo de fase menor que $15^{\circ}$. O K de Fulton foi superior no grupo de peixes de água de esgoto doméstico tratado. A água residuária não apresenta condições para um bom cultivo e um bom desenvolvimento dos peixes, independentemente do uso de probiótico e do método de aferição do estado de saúde utilizado. As cepas probióticas não promoveram melhorias no desempenho desses peixes e nem na sobrevivência.

Palavras-chave: ângulo de fase, desempenho, estresse, impedância

\section{Probiotic in feed for tilapia cultivated in treated domestic sewage effluent}

\begin{abstract}
The bioimpedance is a method used to estimate humans and animals body composition, characterized by determining the value of the phase angle. This study aimed to estimate the fish condition through electrical bioimpedance technique and evaluate the performance of Nile tilapia grown in sewage water treated with the addition of probiotic (Bacillus licheniformis, Bacillus cereus and Bacillus subitillis and yeast - Saccharomyces cerevisiae and boulardi) in diets. For the experiment, 360 fingerlings were used with initial average weight of $2.91 \pm 0.37 \mathrm{~g}$ and initial average length of $3.30 \pm 0.27 \mathrm{~cm}$, distributed in a completely randomized design with three treatments and six repetitions of 20 fish each. Treatments consisted of: PRO1 - fish grown in clean water; RO2 - fish grown in wastewater; PRO3 - fish grown in wastewater + probiotic added to the feed. All animals were fedto apparent satiety. Tilapia that were submitted to the health challenge (wastewater) presented phase angle less than $15^{\circ}$. Fulton's $\mathrm{K}$ value was higher for the group treated with sewage water. The wastewater does not present conditions for a good crop and a good development of the fish, regardless the probiotic supplementation and the measurement method of health status used. The probiotic strains did not led to an improvement of fish performance and survival.
\end{abstract}

Keywords: impedance, performance, phase angle, probiotics, stress 


\section{Introdução}

Problemas relacionados a falta de água estão relacionados principalmente a grande demanda pela agricultura e abastecimento humano. Além disso, ocorre uma distribuição inadequada de recursos hídricos disponível e sua degradação causada pela polvição e pelo mau uso do solo. Diante dessa situação, a procura por mecanismos que proporcionem uma racionalização e reaproveitamento da água, tornam-se essenciais para evitar a polvição dos recursos hídricos.

Trabalhos de pesquisa têm sido desenvolvidos com a utilização da água residuária tratada em diversos setores, inclusive na exploração de peixes, sempre com a perspectiva de (re)aproveitar os recursos hídricos nos empreendimentos, garantindo o desempenho dos animais de forma sustentável (Santos et al., 2009; Santos et al., 2011; Eler \& Millani, 2007).

Ressalta-se que com o crescimento da criação intensiva de peixes, tem-se verificado aumento da pressão de seleção em patógenos, com reflexos na elevação de doenças nos sistemas de produção, principalmente devido à falta de manejo adequado, o que compromete o desempenho e a saúde dos peixes. No entanto, o uso de cepas probióticas na dieta de peixes tem proporcionado melhor aproveitamento dos nutrientes, consequentemente melhores condições do ambiente aquático, além de proporcionar sanidade e segurança alimentar (Nayak, 2010).

A utilização de antibióticos tem sido feita na perspectiva de garantir a saúde de peixes. No entanto, pode promover uma pressão de seleção sobre os microrganismos, promovendo o aumento da resistência bacteriana (Cabello, 2006). Devido a esses problemas relacionados, pesquisas tem sido desenvolvidas com a suplementação de probióticos na dieta, pois são produtos formados de cepas vivas e utilizados como suplemento alimentar. Dessa forma, tem crescido a busca por efeitos benéficos ao hospedeiro, melhorando o equilíbrio da microbiota intestinal, de modo a evitar a proliferação de microrganismos patogênicos (Fuller, 1989).
O método da bioimpedância é utilizado para estimar a condição corporal em humanos e animais, caracterizado pela determinação do valor do ângulo de fase. Quando esse ângulo é superior a $15^{\circ}$, indica que os peixes se encontram em bom estado de saúde, enquanto que os animais que estão com valores inferiores a $15^{\circ}$, estão com sua saúde comprometida, devido a alguma dificuldade promovida pelo ambiente aquícola (Cox \& Heintz, 2009).

A pesquisa foi realizada para estimar a condição dos peixes por meio da mensuração da bioimpedância elétrica (BIA) e avaliar o desempenho de tilápias cultivadas em água de esgoto doméstico tratado com a adição de probiótico (Bacillus licheniformis, Bacillus cereus e Bacillus subitillis e leveduras -Saccharomyces cerevisiae e Saccharomyces boulardi) na ração por 145 dias.

\section{Material e Métodos}

O experimento foi realizado na área experimental da Estação de Tratamento de Esgotos da zona Leste do município de TeresinaPI (ETE-Leste), pertencente à empresa Águas e Esgotos do Piauí S.A. (AGESPISA), no período de outubro de 2011 à março de 2012. A estação é composta de cinco lagoas de estabilização em série, sendo uma facultativa aerada, duas facultativas convencionais (paralelas entre si) e duas de maturação (também paralelas entre si), cujo efluente final foi utilizado no abastecimento dos tanques experimentais.

Foram utilizados 360 alevinos tilápiasdo-nilo (Oreochromis niloticus), durante 145 dias. Os peixes, com peso inicial médio de $2,91 \pm 0,37 \mathrm{~g}$ e comprimento inicial de 3,30 \pm $0,27 \mathrm{~cm}$, foram distribuídos em delineamento inteiramente casualizado, com três tratamentos, seis repetições, sendo a unidade experimental composta por 20 peixes. Os tratamentos consistiram de: $\mathrm{PRO} 1$ - peixes cultivados em água limpa, procedente da rede de abastecimento d'água da AGESPISA (Águas e Esgotos do Piauí S.A) alimentados até a aparente saciedade com ração comercial (10\% da biomassa); PRO2 - peixes cultivados em água residuária (esgoto doméstico tratado por lagoa de estabilização) alimentados até a aparente saciedade com 
ração comercial sem o uso de probiótico; PRO3 - peixes cultivados com água residuária (esgoto doméstico tratado por lagoa de estabilização) alimentados até a aparente saciedade com ração comercial com o uso de probiótico $(1,0$ g. $\left.\mathrm{kg}^{-1}\right)$. Todos os tanques apresentavam um sistema de bombeamento utilizando bolhas de ar emitidas por um soprador radial.

O probiótico utilizado na ração apresentava a seguinte composição: suplemento mineral (PREMIX), farelo de trigo, microrganismos (Bacillus licheniformis, Bacillus cereus e Bacillus subitillis) e leveduras (Saccharomyces cerevisiae e Saccharomyces boulardi). Para a determinação da quantidade de probiótico utilizada na ração experimental determinou-se a proporção de acordo com a biomassa do tratamento avaliado.

O probiótico foi diluído em água com temperatura entre 40 e $45{ }^{\circ} \mathrm{C} \quad(16,67$ 9 de probiótico..-1 de água), conforme recomendação do fabricante. Logo em seguida, foi feita a homogeneização da solução por aproximadamente 30 minutos e borrifou-se o produto na ração, de acordo com a biometria realizada (1,33 $\mathrm{g}$ de probiótico. $\mathrm{kg}^{-1}$ de ração). Deixou-se descansar por 45 minutos antes de sua utilização.

Todos os dias, às sete horas da manhã, o probiótico foi adicionado na ração experimental, apresentando uma estabilidade de até 12 horas. A ração foi dividida em duas refeições durante o dia (8h30min e às 16h).

Utilizou-se uma ração comercial com $32 \%$ de proteína bruta até os 78 dias de idade e outra com $28 \%$ de PB até os 145 dias de vida (Tabela 1), de acordo com a fase de vida dos peixes e realização de biometrias.

Tabela 1. Composição química da ração comercial para tilápias-do-nilo de 1 até os 78 e dos 79 aos 145 dias de idade, com cultivo em efluente de esgoto doméstico tratado

\begin{tabular}{|c|c|c|c|}
\hline $\begin{array}{c}\text { Características nutricionais } \\
\text { da ração até os } 78 \text { dias de } \\
\text { idade? }\end{array}$ & $\begin{array}{c}\text { Valor calculado (g } \\
\text { por } 100 \mathrm{~g} \text { de amostra } \\
\text { úmida) }\end{array}$ & $\begin{array}{c}\text { Características nutricionais } \\
\text { da ração dos } 79 \text { aos } 145 \\
\text { dias de idade }\end{array}$ & $\begin{array}{l}\text { Valor calculado } \\
\text { (g por } 100 \mathrm{~g} \text { de } \\
\text { amostra úmida) }\end{array}$ \\
\hline Umidade (\%) & 9,5 & Umidade (\%) & 9,5 \\
\hline Proteína Bruta (\%) & 32,0 & Proteína Bruta (\%) & 28,0 \\
\hline Extrato Etéreo (\%) & 3,0 & Extrato Etéreo (\%) & 3,0 \\
\hline Fibra Bruta (\%) & 8,0 & Fibra Bruta (\%) & 9,0 \\
\hline Matéria mineral (\%) & 11,0 & Matéria mineral (\%) & 11,0 \\
\hline Cálcio (\%) & 1,8 & Cálcio (\%) & 1,8 \\
\hline Fósforo (\%) & 0,6 & Fósforo (\%) & 0,5 \\
\hline
\end{tabular}

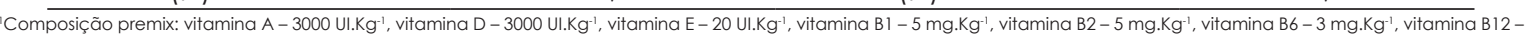
$20 \mathrm{mg} \cdot \mathrm{Kg}^{-1}$, vitamina $\mathrm{C}-200 \mathrm{mg} \cdot \mathrm{Kg}^{-1}$, vitamina $\mathrm{K}-5 \mathrm{mg} \cdot \mathrm{Kg}^{-1}$, niacina $-100 \mathrm{mg} \cdot \mathrm{Kg}^{-1}$, biotina $-0,1 \mathrm{mg} \cdot \mathrm{Kg}^{-1}$, colina $-150 \mathrm{mg} \mathrm{Kg}^{-1}$, ácido fólico -1 mg. $\mathrm{Kg}^{-1}$, ácido pantotênico $-20 \mathrm{mg} \cdot \mathrm{Kg}^{-1}$, cobre $-15 \mathrm{mg} \cdot \mathrm{Kg}^{-1}$, ferro - $100 \mathrm{mg} \cdot \mathrm{Kg}^{-1}$, iodo $-5 \mathrm{mg} \cdot \mathrm{Kg}^{-1}$, manganês - $100 \mathrm{mg} \cdot \mathrm{Kg}^{-1}$, selênio - 0,1 mg.Kg-1zinco - $150 \mathrm{mg} \cdot \mathrm{Kg}^{-1}$, antioxidante - $125 \mathrm{mg} \cdot \mathrm{Kg}^{-1}$. ${ }^{2} \mathrm{Composição} \mathrm{premix:} \mathrm{vitamina}$

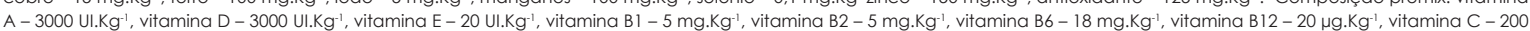
mg. $\mathrm{Kg}^{-1}$, vitamina $\mathrm{K}-5 \mathrm{mg} \cdot \mathrm{Kg}^{-1}$, niacina - $100 \mathrm{mg} \cdot \mathrm{Kg}^{-1}$, biotina $-0,52 \mathrm{mg} \cdot \mathrm{Kg}^{-1}$, colina $-800 \mathrm{mg} \cdot \mathrm{Kg}^{-1}$, ácido fólico $-4,8 \mathrm{mg} \cdot \mathrm{Kg}^{-1}$, cobre $-15 \mathrm{mg} \cdot \mathrm{Kg}^{-1}$, ferro - $100 \mathrm{mg} \cdot \mathrm{Kg}^{-1}$, iodo - $5 \mathrm{mg} \cdot \mathrm{Kg}^{-1}$, manganês - $100 \mathrm{mg} \cdot \mathrm{Kg}^{-1}$, selênio $-0,1 \mathrm{mg} \cdot \mathrm{Kg}^{-1}$, zinco $-150 \mathrm{mg} \cdot \mathrm{Kg}^{-1}$, antioxidante $-125 \mathrm{mg} \cdot \mathrm{Kg}^{-1}$.

Diariamente foram renovados $20 \%$ do volume da água de esgoto tratada em todos os tanques. A aeração foi realizada operando em um turno de aeração de nove horas, funcionando no período das 21 às 6 horas.

Foram avaliados os parâmetros em cada uma das repetições dos tratamentos para a determinação do: $\mathrm{pH}$ e temperatura $\left({ }^{\circ} \mathrm{C}\right)$ pelo método eletrométrico e um equipamento (pHmetro - WTH pH 330i); condutividade elétrica $\left(\mu \mathrm{s} . \mathrm{cm}^{-1}\right)$ pelo método eletrométrico e equipamento (mulímetro/WTW - INOLAB MULTI 720); oxigênio dissolvido (mg. $\mathrm{L}^{-1}$ ) pelo método eletrométrico e equipamento (oxímetro - HANNA - HI 9146); amônia (mg.L-1) pelo método espectro/nesselerização direta e equipamento (Espectofotômetro DR 2800 $\mathrm{HACH}$ ); nitrito (mg. $\mathrm{L}^{-1}$ ) pelo método espectro/ sulfanilamida/ethilenodiamina e equipamento (espectrofotométrico/persufalto de amônia e equipamento (Espectofotômetro DR 2800 $\mathrm{HACH}$ ); fósforo total (mg. $\left.\mathrm{L}^{-1}\right)$ pelo método espectofotométrico/ persulfato de amônia (Espectofotômetro DR $2800 \mathrm{HACH}$ ); demanda bioquímica de oxigênio (DBO), conforme descrito em APHA (2005), sendo feita em frascos padrão e incubadora de DBO; demanda química de oxigênio (DQO), pelo método da refluxação fechada do dicromato com o uso do bloco digestor CIENLAB Dry Block; coliformes, 
pelo método Colilert por meio da seladora QalliTray/estufa/lâmpada UV; clorofila-a, pelo método de Jones, com extração a quente com metanol $100 \%$ e equipamento (Espectofotômetro DR $2800 \mathrm{HACH}$ ); algas, com amostras de água sendo coletadas para visualização do tipo de alga presente por meio de um microscópio binocular com capacidade de $10 \times 100$; e ovos de helmintos, em que a água coletada foi analisada pelo método de sedimentação simples de Hoffmann (1934) para pesquisa de ovos de trematódeos, cestódeos e cistos de Entamoeba histolytica, em que foram observadas cinco laminas de cada amostra e pelo método de Willis (1921) para pesquisa de ovos de nematódeos e oocistos de protozoários. Todas estas observações foram realizadas em microscópio de luz com objetivas 10 e 40X.

Foram calculados para cada parcela experimental as variáveis de desempenho e sobrevivência, de acordo com as equações: 1 - ganho de peso diário $(\mathrm{GPD})=(\mathrm{Pf}-\mathrm{Pi}) / \mathrm{T} ; 2$ - consumo diário de ração $(C D R)=C R / T ; 3$ conversão alimentar aparente $(C A A)=C R / G P$; 4 - taxa de crescimento específico (TCE) $=[(\operatorname{lnPf}$ $-\ln \mathrm{Pi}) \times 100] / \mathrm{T}$ e 5 - Sobrevivência $(\mathrm{S})=100 *\left(\mathrm{~N}^{\circ}\right.$ inicial de peixes $-\mathrm{N}^{\circ}$ final de peixes) $/ \mathrm{N}^{\circ}$ inicial de peixes. Em que: : $\mathrm{Pf}$ - peso final; $\mathrm{Pi}$ - peso inicial; T - tempo experimental; GP - ganho de peso; CR - consumo de ração.

Ao término do experimento foi realizado o teste de bioimpedância elétrica em todos os peixes das unidades experimentais com 0 propósito de determinar o ângulo de fase (AF). Todos os peixes foram inicialmente anestesiados em solução de óleo de cravo (solução estocada: $5 \mathrm{~mL}$ eugenol + $95 \mathrm{~mL}$ álcool etílico p.a), com 2 $\mathrm{mL}$ da solução para cada litro de água. Após a observação do decúbito lateral e redução dos movimentos operculares, determinou-se o peso, comprimento total e o comprimento padrão, para seguir com o procedimento padrão da mensuração da bioimpedância. Para isto, pares de agulhas hipodérmicas (Delta 20-5) fixadas em suporte de acrílico foram aplicadas láterodorsalmente, em pontos de áreas musculosas dos peixes, suavemente enxutos com toalha comum (tecido de algodão). A profundidade da penetração foi de aproximadamente 1,0 $\mathrm{cm}$ e os pontos de aplicação, rigorosamente definidos, foram adaptados do procedimento de Willis \& Hobday (2008).

Os eletrodos injetores de corrente foram conectados a agulhas aplicadas nos peixes (Figura 1), de acordo com Andrade et al. (2014), sendo o ponto de inserção anterior localizado medialmente a um segmento de reta transversal que vai da linha dorsal à linha mediana e que tangencia posteriormente o opérculo e o outro ponto posterior localizado medialmente em um segmento de reta transversal que vai da linha dorsal à linha mediana e que tangencia anteriormente a inserção da nadadeira anal. Os eletrodos detectores (eletrodos internos ou medidores da resistência e reatância) foram aplicados entre os injetores de corrente, a $1 \mathrm{~cm}$ de cada.

Uma vez inseridas as agulhas no animal anestesiado e conectadas aos eletrodos do plessímetro tetrapolar (modelo BIA-101Q, RJL Systems. Clinton Township, Ml, USA, http://www. rjlsystems.com), foi aplicada a corrente alternada $(800 \mu \mathrm{A}$ e $50 \mathrm{KHz})$, por meio dos eletrodos externos (injetores de corrente). Os eletrodos internos (detectores de corrente) possibilitaram a medição direta das variáveis $R$ (resistência) e Xc (reatância). A bioimpedância (Z), o ângulo de fase (ÂF), e o índice de composição (IC $\left.C_{E E}\right)$ foram posteriormente calculados, conforme a fórmula: $\mathrm{AF}=$ [arco-tangente $(\mathrm{XC} / \mathrm{R})] \times\left(180^{\circ} / \pi\right)$ e Z $=\left(R^{2}+X C^{2}\right)^{1 / 2}$ (Barbosa Silva, 2003).

Após a realização da bioimpedância elétrica, os peixes foram devolvidos para um tanque com capacidade de 500 L com água renovável para recuperação da anestesia, observando-se o retorno dos movimentos operculares adequados e sua mobilidade espontânea. Em seguida os peixes foram devolvidos para os seus respectivos tratamentos e repetições.

Todos os resultados foram submetidos à análise da variância, sendo feito o teste de normalidade dos dados, usando o teste de Shpairo-Wilk, teste de homecedasticidade (igualdade de variância) usando o teste de Brown Forsythe e as médias foram comparadas pelo teste de Tukey com $\alpha=0,05$, de acordo com os Proc GLM do STATISTICAL ANALYSIS SYSTEM. 


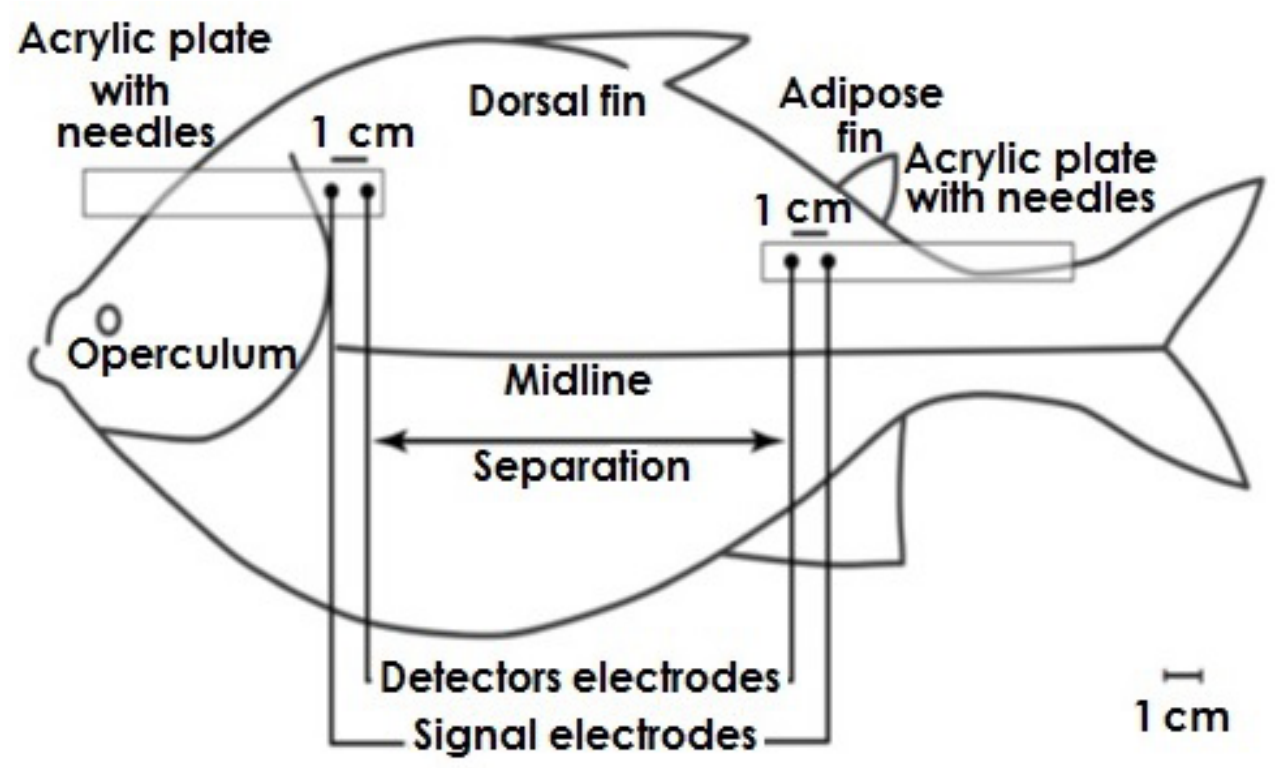

Figura 1. Pontos de inserção de agulhas para serem inseridos os eletrodos injetores de corrente, conforme Andrade et al. (2014).

O projeto de pesquisa intitulado de: "Utilização de probiótico na alimentação de tilápias (Oreochromis niloticus) cultivadas em efluentes de esgotos doméstico tratado", foi submetido ao Comitê de Ética e Pesquisa da Universidade Federal do Piauí sob número de protocolo 013/12 e obteve parecer aprovado (Carta de aprovação n0 $011 / 12$ ).

\section{Resultados e Discussão}

A condutividade elétrica, $\mathrm{DBO}_{5,20^{\prime}}$ fósforo total, coliformes (totais e termotolerantes), clorofila-a e amônia total, constatou-se que os valores observados (Tabela 2) foram superiores $(\mathrm{P}<0,05)$ na água de esgoto doméstico tratado com e sem probiótico, fato que caracteriza desafio sanitário (análise da qualidade da água residuária) para os peixes submetidos a esses tratamentos.

A concentração de coliformes totais nos tratamentos da água de esgoto doméstico tratado encontra-se superior ao preconizado pela resolução 357 do Conama (2005) para o efluente a ser utilizado na aquicultura (5000 NMP.100 mL-1). Essa elevada concentração pode comprometer o desempenho dos peixes, assim como influenciar o consumo pelo homem devido a elevada carga de coliformes presentes. O mesmo foi constatado na concentração de coliformes termotolerantes que estabelece limite de 1000 NMP.100 mL-1. Ou seja, pela resolução, esse efluente não pode ser utilizado no cultivo intensivo de peixes, pois pode comprometer o desempenho dos animais, assim como a saúde da população, conforme as variáveis avaliadas na Tabela 3.

O excesso de matéria orgânica em decomposição provido das lagoas de estabilização, promoveram uma elevada condutividade elétrica $\left(\mu s . \mathrm{cm}^{-1}\right), \quad$ o que comprometeu o desempenho dos peixes (Tabela 3). Neste sentido, Dias et al. (2011), ao avaliarem o desempenho de larvas de matrinxã com o uso de probiótico composto de Bacillus subtilis, encontraram baixos valores para o parâmetro condutividade elétrica $\left(203,13 \mu \mathrm{s} . \mathrm{cm}^{-1}\right)$ indicando que o desafio sanitário oriundo da fonte de água não foi suficiente para a expressão do efeitos do probiótico.

Para Colt (2006), a grande concentração de amônia total encontrada no ambiente aquícola pode acarretar mortalidade e queda no desempenho dos animais. Os tratamentos com água residuária indicam elevada decomposição, o que torna o ambiente desfavorável aos peixes. Com relação a $\mathrm{DBO}_{5,20^{\prime}}$ a faixa ideal se encontra entre 30 e $50 \mathrm{mg} \cdot \mathrm{L}^{-1}$. Os animais que estavam nos tratamentos com água de esgoto doméstico tratado apresentaram valores superiores, fato que evidencia ambiente desafiador para os peixes, em virtude da avalição da qualidade da água. A resolução 357 do Conama (2005) afirma 
que para o lançamento do efluente em águas de classe 2 a quantidade de DBO deve ser inferior a 5 mg.L.-1. Logo, a concentração encontrada nos tratamentos com a água de esgoto doméstico tratado encontra-se bem superior ao limite estabelecido pela resolução, não sendo indicado para a aquicultura, pois compromete o desempenho dos peixes (Tabela 3).

Tabela 2. Valores médios de parâmetros ambientais da qualidade da água da produção de tilápias durante 145 dias de cultivo, em função dos tratamentos experimentais

\begin{tabular}{|c|c|c|c|}
\hline Parâmetros & $\begin{array}{c}\text { Água limpa sem } \\
\text { probióticos }\end{array}$ & $\begin{array}{c}\text { Água residuária sem } \\
\text { probióticos }\end{array}$ & $\begin{array}{l}\text { Água residuária } \\
\text { com probióticos }\end{array}$ \\
\hline & \multicolumn{3}{|c|}{ Médias \pm desvio padrão } \\
\hline Cond. elét. $\left(\mu \mathrm{s} . \mathrm{cm}^{-1}\right)$ & $67,8 \pm 10,13^{b}$ & $406,0 \pm 126,42^{a}$ & $475,1 \pm 38,73^{a}$ \\
\hline $\mathrm{DBO}_{5,20}\left(\mathrm{mg}^{\left.-\mathrm{L}^{-1}\right)}\right.$ & $31,7 \pm 12,66^{b}$ & $74,4 \pm 59,49 a$ & $50,4 \pm 13,19 a$ \\
\hline $\mathrm{DQO}\left(\mathrm{mg} \cdot \mathrm{L}^{-1}\right)$ & $69,1 \pm 18,04^{a}$ & $80,5 \pm 17,91^{a}$ & $80,9 \pm 16,92^{a}$ \\
\hline$O D\left(m g . L^{-1}\right)$ & $6,3 \pm 0,81^{a}$ & $3,2 \pm 0,41^{b}$ & $2,6 \pm 0,55^{b}$ \\
\hline $\mathrm{pH}$ & $8,3 \pm 0,8^{a}$ & $7,8 \pm 0,13^{a}$ & $7,9 \pm 0,11^{a}$ \\
\hline Fósforo total (mg. $\left.L^{-1}\right)$ & $1,7 \pm 0,37^{b}$ & $3,4 \pm 0,50^{a}$ & $3,9 \pm 0,50^{a}$ \\
\hline Nitrito (mg. $\left.L^{-1}\right)$ & $0,26 \pm 0,14^{a}$ & $0,25 \pm 0,23^{a}$ & $0,34 \pm 0,27^{a}$ \\
\hline Amônia total (mg.L-1) & $1,60 \pm 1,58^{b}$ & $19,36 \pm 11,04^{a}$ & $22,91 \pm 7,91^{a}$ \\
\hline Temperatura $\left({ }^{\circ} \mathrm{C}\right)$ & $26,5 \pm 0,15^{a}$ & $26,8 \pm 0,14^{a}$ & $27,0 \pm 0,09 a$ \\
\hline Clorofila-a $\left(\mu \mathrm{g} \cdot \mathrm{L}^{-1}\right)$ & $428,9 \pm 71,69^{b}$ & $745,0 \pm 61,79 a$ & $681,6 \pm 76,03^{a}$ \\
\hline Coliformes totais (NMP.100 mL-1) & $4,74 \mathrm{E}+03 \pm 9,28 \mathrm{E}+02^{\mathrm{b}}$ & $1,62 \mathrm{E}+04 \pm 4,29 \mathrm{E}+03^{a}$ & $3,52 E+04 \pm 6,37 E+03^{a}$ \\
\hline Coliforme termotolerante (NMP.100 $\mathrm{mL}^{-1}$ ) & $1,15 E+01 \pm 3,92 E+01^{b}$ & $1,43 \mathrm{E}+03 \pm 3,13 \mathrm{E}+02^{a}$ & $3,56 \mathrm{E}+03 \pm 3,86 \mathrm{E}+02^{a}$ \\
\hline Ovos de helmintos & ND & ND & ND \\
\hline
\end{tabular}

Tabela 3. Valores médios das variáveis de desempenho zootécnico e taxa de sobrevivência de tilápias até os 145 dias de cultivo, em função dos tratamentos experimentais.

\begin{tabular}{lccc}
\hline Variáveis & Água limpa sem probióticos & $\begin{array}{c}\text { Água residual sem } \\
\text { probióticos }\end{array}$ & $\begin{array}{c}\text { Água residual com } \\
\text { probióticos }\end{array}$ \\
\hline Peso final (g.peixe-1) & $395,69 \pm 47,73^{a}$ & $184,86 \pm 53,36^{\mathrm{b}}$ & $150,59 \pm 41,23^{\mathrm{b}}$ \\
Consumo total de ração (g) & $17200,44 \pm 710,02^{a}$ & $5666,75 \pm 279,922^{\mathrm{b}}$ & $5101,99 \pm 280,87^{\mathrm{b}}$ \\
CAA & $1,17 \pm 0,16^{\mathrm{a}}$ & $1,48^{\mathrm{a}} \pm 0,40^{\mathrm{a}}$ & $1,21 \pm 0,34^{\mathrm{a}}$ \\
TCE (\%) & $3,22 \pm 0,08^{\mathrm{a}}$ & $2,64 \pm 0,27^{\mathrm{b}}$ & $2,75 \pm 0,25^{\mathrm{b}}$ \\
Sobrevivência (\%) & $91,67^{\mathrm{a}}$ & $57,5^{\mathrm{b}}$ & $62,5^{\mathrm{b}}$ \\
\hline ab.bédias seguidas de letras iguais na linha não diferem estatisticamente pelo teste de Tukey a 5\% de significância (P>0,05).
\end{tabular}

Não foram encontradas diferenças significativas $(P>0,05)$ nos parâmetros $D Q O$, $\mathrm{pH}$, nitrito e temperatura (Tabela 2). Os valores de DQO encontram-se bem abaixo da concentração média típica para regiões de clima quente que é de $400 \mathrm{mg} \cdot \mathrm{L}^{-1}$. Segundo a resolução 357 do Conama (2005), a concentração de DQO deve ser inferior a 450 mg. $\mathrm{L}^{-1}$ para o seu uso na aquicultura, sendo que nos tratamentos com a água de esgoto doméstico tratado foram constatados valores inferiores. Já $\mathrm{p} \mathrm{pH}$ da água nas unidades experimentais estava dentro da faixa ideal, entre 6,5 a 9,0 (Zhou et al., 2009). Mehrim (2009) afirma que para as cepas probióticas terem efeitos desejáveis no trato digestivo dos peixes é necessário que os fatores ambientais, dentre eles $\mathrm{o} \mathrm{pH}$, estejam funcionando de maneira adequada, por meio de uma boa qualidade de água. A concentração de nitrito encontrada nos tratamentos está bem abaixo da faixa compreendida entre 0,7 e 2,0 mg. $\mathrm{L}^{-1}$. Normalmente, no cultivo de peixes em sistemas intensivos, ocorre elevada concentração de nitritos, devido à transformação que acontece por meio da atuação de bactérias do gênero Nitrosomonas, da amônia livre em nitritos (Kubitza, 2000). Este aspecto não foi detectado no experimento. No entanto, o que promove a dificuldade do ambiente aquícola aos peixes é um conjunto de fatores que quando interrelacionados promovem queda no desempenho e até mesmo provoca sua mortalidade.

Para o cultivo de peixes tropicais a temperatura nos tratamentos avaliados está dentro das recomendações que é entre 18 a 30 ${ }^{\circ} \mathrm{C}$ (Kubitza, 2000). Uma temperatura média de 28 ${ }^{\circ} \mathrm{C}$ é considerada ótima para o cultivo de tilápias- 
do-nilo, em sistema de recirculação forçada (ElSayed \& Kawanna, 2008). Marengoni et al. (2010), ao avaliarem o desempenho e proporção sexual de tilápia vermelha com inclusão de probiótico em água mesohalina, também encontraram temperatura média de $28,5^{\circ} \mathrm{C}$. Temperaturas muito elevadas podem proporcionar diminuição no desempenho dos peixes, já que seu aumento causa gasto energético excessivo para manter o funcionamento do organismo.

Constatou-se que a concentração de oxigênio dissolvido e clorofila-a (Tabela 2) foram superiores $(P<0,05)$ no tratamento com água limpa. A faixa ideal de oxigênio dissolvido é de 5 a-8 mg.L-1. (Kubitza, 2000). Pela resolução 357 do Conama (2005), a concentração de OD deve ser superior a 5,0 mg.L-1 para o efluente ser utilizado na aquicultura. Sendo assim, os tratamentos com a água de esgoto doméstico tratado estavam foram deste padrão para o efluente de classe de 2 e representa um ambiente extremamente desafiador aos animais. Mesmo, com a utilização de aeradores nos tratamentos com água tratada de esgoto doméstico, estes aeradores não foram suficientes para deixar o teor de OD dentro da faixa ótima para o cultivo da espécie. Tran-duy et al. (2008) reduziram as concentrações de oxigênio dissolvido no cultivo de tilápias-do-nilo e detectaram queda no desempenho devido ao desafio pela baixa concentração de OD no meio.

O tratamento com água limpa apresentou elevada concentração de clorofila-a, indicando assim uma elevada produtividade primária do meio. Assim, com base nos parâmetros físico-químicos, avaliados durante todo o período experimental, fica evidenciado que a água tratada de esgoto doméstico representa um ambiente de desafio aos peixes.

O peso final, o consumo de ração, a taxa de crescimento específico e a sobrevivência (Tabela 3) apresentaram diferenças significativas $(P<0,05)$, sendo observado valores médios maiores no tratamento de água limpa. A carga orgânica presente na água residuária promoveu queda no desempenho das tilápias conforme variáveis apresentadas. Os resultados para peso, comprimento e sobrevivência estão de acordo com os encontrados por MEURER et al. (2007), ao testarem a inclusão de $S$. cerevisiae em rações para tilápia-do-Nilo A conversão alimentar aparente não apresentou efeito significativo $(P>0,05)$ entre os tratamentos. NAVARRO et al. (2010) encontraram valores similares da taxa de crescimento específico em tilápias-do-Nilo $(4,0 \%)$ com suplementação de vitamina $C$, no entanto, os peixes não foram submetidos a desafio sanitário.

As cepas probióticas utilizadas nesse experimento não surtiram efeito nas variáveis de desempenho, sendo o grupo de tilápias que se alimentavam da ração que continha o probiótico não se equiparou aos peixes cultivados na água residuária sem o seu uso. Foi constatada uma maior sobrevivência (Tabela 3) nos peixes cultivados na água limpa, comprovando o efeito desafiador e comprometedor de desempenho de tilápias cultivadas em água residuária, independente do uso ou não de probiótico na ração, apresentando uma elevada mortalidade e promovendo dificuldades para o desenvolvimento dos animais.

Observou-se que a reatância (Tabela 4) não foi influenciada $(P>0,05)$ pelos tratamentos avaliados durante a fase adulta de tilápias-donilo. Porém, constatou-se para os parâmetros: ângulo de fase, peso, comprimento padrão, comprimento, índice de composição e fator de condição de Fulton, que os valores foram superiores para os tratamentos com água limpa $(P<0,05)$, enquanto os com água residuária com e sem probióticos não diferiram entre si $(P>0,05)$. Os valores para impedância e resistência (Tabela 4) do tratamento com água limpa foram inferiores $(P<0,05)$ aos com os de água residuária com e sem probiótico, que não diferiram entre si $(P>0,05)$.

O AF é utilizado como uma nova ferramenta para a mensuração do estado de saúde dos peixes, assim como de outros animais. Cox \& Heintz (2009), ao realizarem trabalhos em peixes para a avaliação da condição de saúde dos animais, com base na determinação do ângulo de fase, em testes de bioimpedância elétrica (BIA), verificaram que ângulos de fase maiores que $15^{\circ}$ indicam boa condição do peixe. 
Tabela 4. Valores médios do ângulo de fase $(A F)$, impedância $(Z)$; reatância $(X C)$; resistência $(R)$; peso $(P)$; comprimento padrão (CP); distância dos eletrodos (DDi); comprimento (L); índice de composição (IC) e fator de condição Fulton (K de Fulton) de tilápias-do-nilo, até os 145 dias de cultivo, em função dos tratamentos

\begin{tabular}{|c|c|c|c|}
\hline Parâmetros & $\begin{array}{l}\text { Água limpa sem } \\
\text { probióticos }\end{array}$ & $\begin{array}{c}\text { Água residuária sem } \\
\text { probióticos }\end{array}$ & $\begin{array}{l}\text { Água residuária } \\
\text { com probióticos }\end{array}$ \\
\hline & \multicolumn{3}{|c|}{ Médias \pm desvio padrão } \\
\hline $\mathrm{AF}$ & $16,00 \pm 0,76^{a}$ & $14,40 \pm 1,14^{b}$ & $14,14 \pm 0,64^{b}$ \\
\hline Z & $142,74 \pm 5,05^{b}$ & $155,71 \pm 12,51^{\mathrm{ab}}$ & $159,13 \pm 8,85^{a}$ \\
\hline$X_{c}$ & $39,37 \pm 2,97^{a}$ & $38,54 \pm 1,37^{a}$ & $38,75 \pm 1,6^{a}$ \\
\hline$R$ & $137,18 \pm 4,47^{b}$ & $150,83 \pm 12,77^{a b}$ & $154,31 \pm 8,88^{a}$ \\
\hline$P(g)$ & $431,62 \pm 55,37^{a}$ & $186,71 \pm 88,73^{b}$ & $155,95 \pm 68,59^{b}$ \\
\hline $\mathrm{CP}(\mathrm{cm})$ & $22,80 \pm 1,87^{a}$ & $17,33 \pm 2,42^{\mathrm{b}}$ & $16,20 \pm 2,14^{\mathrm{b}}$ \\
\hline DDi (cm) & $3,03 \pm 1,03^{b}$ & $6,45 \pm 1,15^{a}$ & $6,21 \pm 1,34^{a}$ \\
\hline $\mathrm{L}(\mathrm{cm})$ & $27,19 \pm 1,83^{a}$ & $21,3778 \pm 2,92^{b}$ & $20,1386 \pm 2,65^{b}$ \\
\hline IC & $2,56 \pm 0,3620^{a}$ & $1,1633 \pm 0,43^{b}$ & $1,0540 \pm 0,49^{b}$ \\
\hline K de Fulton & $2,12 \pm 0,08^{a}$ & $1,7867 \pm 0,14^{b}$ & $1,7880 \pm 0,08^{b}$ \\
\hline
\end{tabular}

No entanto, quando esses valores são menores que $15^{\circ}$, caracterizam péssimo estado de saúde dos animais. Observou-se que os peixes que foram submetidos à água de esgoto doméstico tratado apresentaram ângulo de fase (Tabela 4) menor que $15^{\circ}$, indicando assim, pior condição no estado de saúde dos animais, devido ao ambiente aquícola. Já as tilápiasdo-nilo que foram submetidas ao tratamento com água limpa obtiveram AF superior a $15^{\circ}$, indicando que os peixes estavam em melhor estado de saúde. Este resultado é provocado pela qualidade da água, em que os animais estavam inseridos.

A impedância apresentou valores superiores nos peixes que estavam presentes na água residuária, sendo que essa variável se refere justamente ao impedimento ou a oposição das barreiras celulares a algo que pode estar comprometendo o desempenho ou o próprio bem-estar animal. Isso confirma o ambiente extremamente desafiador aos peixes $e$ que o organismo buscava soluções para superar a péssima qualidade de água presente. Dessa forma, o ângulo de fase impedância permite avaliar o estado fisiológico das membranas celulares e sua participação da distribuição da água extra e intracelular. Destaca-se que essas variáveis têm sido realizadas em operações de rastreamento e etiquetagem devido a impossibilidade de medição direta do estado de saúde dos peixes (Bradford et al., 2009).

Mesmo os animais, que foram submetidos ao tratamento com a água residuária e a adição de probiótico na ração, não apresentaram melhor ângulo de fase quando comparado ao tratamento com água residuária e sem probiótico $(P>0,05)$.

Os peixes que se encontravam sob desafio sanitário intenso, assim como verificado com os peixes que se encontravam na água de esgoto doméstico tratado apresentaram um $\mathrm{K}$ de Fulton inferior ao tratamento de água limpa, tornando-se um forte indicador do estado de saúde dos animais. Andrade et al. (2014), avaliando esses parâmetros na espécie tambatinga, observaram que estas variáveis não modificaram em animais durante 50 dias de crescente e paulatina eutrofização, em níveis subletais, demonstrando adaptação dos peixes ao ambiente aquícola adotado.

Ressalta-se que na condução do experimento os eletrodos foram inseridos na parte dorsal, para manter o mesmo tipo de célula avaliada, e as distâncias entre os eletrodos apresentou pequenos desvios, justamente para evitar variações bruscas durante a leitura do aparelho de bioimpedância, fato esse constatado por Cox et al. (2011). Ressalta-se que a importância de que mudanças relacionadas às distâncias dos eletrodos e do posicionamento (lado dorsal e ventral) podem proporcionar leituras diferentes devido ao tipo de tecido avaliado (Cox et al., 2011). A determinação do melhor local para a inserção dos eletrodos nas tilápias-do-nilo é essencial para a representação da BIA como forma de apresentação global do corpo de animal.

A bioimpedância elétrica pode ser considerada uma importante ferramenta de 
mensuração do estado de saúde dos animais já que não precisa sacrificar os animais tornando-se uma ferramenta essencial para produtores de peixes. Na pesca pode ser feito - acompanhamento de uma determinada população de peixes ao longo do tempo e ir observando o sev estado de saúde. A avaliação de um grupo de animais apenas pelo peso é considerada uma prática insegura pois ocorre variações nas interpretações de quem está avaliando (Helfman, 2007).

O IC apresentou valores inferiores nas tilápias cultivadas em água residuária, indicando que os animais perderam peso, o que está confirmado pela averiguação do peso dos animais, devido justamente a elevada carga orgânica presente na água.

Os cuidados na piscicultura devem ser dobrados já que pequenos descuidos podem comprometer um lote inteiro de animais. Mudanças no estado nutricional dos animais compromete o seu desempenho, causando prejuízos não só para o grupo de peixes como também para o produtor (Zaniboni-Filho \& Nuñer, 2004).

\section{Conclusões}

A água residuária não apresenta condições para um bom cultivo e um bom desenvolvimento dos peixes, independentemente do uso de probiótico e do método de aferição do estado de saúde utilizado. As cepas probióticas não promoveram melhorias no desempenho desses peixes e nem na sobrevivência.

\section{Referências}

Andrade, F.T., Abreu, M.L.T., Lopes, J.B., Figueiredo, A.V., Araripe, M.N.B.A., Ferreira, A.H.C. 2014. Ichthyometry and electrical bioimpedance analysis to estimate the body composition of tambatinga. Acta Amazonica 44: 279-286.

Barbosa Silva, M.C.G. 2003. Can Bioeletrical Impedance Analysis Identify Malnutrition in Preoperative Nutrition Assessment. Nutrition 19: 422-426.

Bradford, R.W., Hobday, A.J., Evans, K., Lansdell, M. 2009. CMAR Code of Practice for Tagging Marine Animals. CSIRO Marine and Atmospheric Research Paper 028. CSIRO, Hobart, 36p.
Cabello, F.C. 2006. Heavy use of prophylactic antibiotics in aquaculture: a growing problem for human and animal health and for the environment. Environment Microbiology 8: 1137 1144.

Colt, J. 2006. Water quality requirements for reuse systems. Aquacultural Enginnering 34: 143- 56.

CONAMA. Resolução CONAMA 357/2005. Dispõe sobre a classificação dos corpos de água e diretrizes ambientais para o seu enquadramento, bem como estabelece as condições e padrões de lançamento de efluentes, e dá outras providências. 2005. http://www.mma.gov.br/ port/conama/legiabre. <Acesso em 10 Fev . 2013> cfm? codlegi $=459 /$

Cox, M.K., Heintz, R. 2009. Electrical phase angle as a new method to measure fish condition. Fishery Bulletin 107: 477-487.

Cox, M.K., Heintz, R., HARTMAN, K. 2011. Measurements of resistance and reactance in fish with the use of bioelectrical impedance analysis: sources of error. Fishery Bulletin 109: 34-47.

Dias, D.D.C., Corrêa, C.F., Leonardo, A.F.G., Tachibana, L., Romagosa, E., Paiva, M.J.T.R. 2011. Probiótico na larvicultura de matrinxã, Brycon amazonicus. Acta Scientiarum Animal Sciences 33: 365-368.

Eler, M.N., Millani, T.J. 2007. Métodos de estudos de sustentabilidade aplicados à aquicultura. Revista Brasileira de Zootecnia 36: 33-44.

El-Sayed, A.F.M. 2006. Tilapia culture. 1.ed. CABI Publishing, Cambridge, EUA. 277p

El-Sayed, A.F.M., Kawanna, M. 2008. Optimum water temperature boosts the growth performance of Nile tilapia (Oreochromis niloticus) fry reared in a recycling system. Aquaculture Research 39: 670-672.

Fuller, R. 1989. A review: probiotic in man and animals. Journal Applied Environmental Microbiology 63: 1034-1039.

Helfman, G.S. 2007. Fish conservation: A guide to understanding and restoring global aquatic biodiversity and fisheries resource. Island Press, Washington, EUA. 584p.

Kubitza, F. 2000. Tilápia - tecnologia e planejamento na produção comercial. Divisão de Biblioteca e Documentação, Jundiaí, Brasil. 289p.

Marengoni, N.G., Albuquerque, D.M., Mota, F.S. 2010. Desempenho e proporção sexual de tilápia vermelha sob à inclusão de probiótico em água mesohalina. Archivos de Zootecnia 59, 403-414. 
Mehrim, A.I.2009.Effectofdietarysupplementation of Biogen (Commercial probiotic) on mono-sex Nile tilapia Oreochromis niloticus under different stocking densities. Journal Fish Aquatic Scince 4: 261-273.

Meurer, F., Hayashi, C., Costa, M.M. 2007. Saccharomyces cerevisiae como probiótico para alevinos de tilápia-do-nilo submetidos a desafio sanitário. Revista Brasileira de Zootecnia 36: 1219-1224.

Navarro, R. D., Ferreira, W. M., Ribeiro Filho, O. P., Botion, L. M., Pereira, F. K. S., Silva, R. F., Maciel, T. E. F. 2010. Desempenho de tilápia do Nilo (Oreochromis niloticus) suplementada com vitamina C. Archivos de Zootecnia 59: 589-596.

Nayak, S.K. 2010. Probiotics and immunity: A fish perspective. Fish \& Shelfish Immunology, 29: 2-14.

Santos, E.S., Furtado-Neto, M., Mota, S., Dos Santos, A.B., Aquino, M.D. 2009. Cultivo de tilápias-do-nilo em esgoto doméstico tratado, com diferentes taxas de alimentação. Revista DAE 180: 4-11.

Santos, E.S., Mota, S., Santos, A.B., Monteiro, C.A.B., Fontenele, R.M.M. 2011. Avaliação da sustentabilidade ambiental do uso de esgoto doméstico tratado na piscicultura. Engenharia Sanitária e Ambiental 16: 45-54.

Tran-Duy, A, Schrama, J.W., Vam Dam. A.A., Verreth, J.A.J. 2008. Effects of oxygen concentration and body weight on maximum feed intake, growth and hematological parameters of Nile tilapia, Oreochromis niloticus. Aquaculture 275: 152-162.

Willis, J, Hobday, A.J. 2008. Application of bioelectrical impedance analysis as a method for estimating composition and metabolic condition of southern bluefin tuna (Thunnus maccoyii) during conventional tagging. Fisheries Research 93: 64-71.

Zaniboni-Filho, E., Nuñer, A.P. de O. 2004. Fisiologia da reprodução e propagação artificial dos peixes. Tópicos especiais em piscicultura de água doce tropical intensiva. TecArt 1: 45-73.

Zhou, Q., Li, K., Jun, X., Bo, L. 2009. Role and functions of beneficial micro-organisms in sustainable aquaculture. Bioresource Technology 100: 3780-3786. 